\title{
Culture of salivary methanogens assisted by chemically produced hydrogen.
}

2

3 Guindo $\mathrm{CO}^{1,2}$, Terrer $\mathrm{E}^{2,3}$, Chabrière $\mathrm{E}^{2}$, Aboudharam $\mathrm{G}^{2,3}$, Drancourt $\mathrm{M}^{2^{*}}$, Grine $\mathrm{G}^{1,2,3}$.

5 1. IHU Méditerranée Infection, Marseille, France.

6 2. Aix-Marseille Univ., IRD, MEPHI, IHU Méditerranée Infection, Marseille, France.

7 3. Aix-Marseille-Univ., Faculty of Odontology, Marseille, France.

8

9

10 *Corresponding author: Prof. Michel Drancourt

11 IHU Méditerranée Infection, UMR MEPHI

12 19-21, Bd Jean Moulin 13005 Marseille, France. Tel: +33 (0)4 1373 24 01, fax: + 33 (0) 13

$13 \quad 732402$.

14 Email : michel.drancourt@univ-amu.fr. 


\section{ABSTRACT}

16 Methanogen cultures require hydrogen produced by fermentative bacteria such as

17 Bacteroides thetaiotaomicron (biological method). We developed an alternative method for

18 hydrogen production using iron filings and acetic acid with the aim of cultivating

19 methanogens more efficiently and more quickly (chemical method). We developed this new

20 method with a reference strain of Methanobrevibacter oralis, compared the method to the

21 biological reference method with a reference strain of Methanobrevibacter smithii and finally

22 applied the method to 50 saliva samples. Methanogen colonies counted using ImageJ

23 software were identified using epifluorescence optical microscopy, real-time PCR and PCR

24 sequencing. For cultures containing the pure strains of M. oralis and M. smithii, colonies

25 appeared three days postinoculation with the chemical method versus nine days with the

26 biological method. The average number of $M$. smithii colonies was significantly higher with

27 the chemical method than with the biological method. There was no difference in the delay of

28 observation of the first colonies in the saliva samples between the two methods. However, the

29 average number of colonies was significantly higher when using the biological method than

30 when using the chemical method at six days and nine days postinoculation (Student's test, $\mathrm{p}=$

310.005 and $\mathrm{p}=0.04$, respectively). The chemical method made it possible to isolate four

32 strains of $M$. oralis and three strains of M. smithii from the 50 saliva samples. Establishing

33 the chemical method will ease the routine isolation and culture of methanogens. 


\section{INTRODUCTION}

37 Methanogenic archaea (referred to herein as methanogens) are acknowledged members of the

38 digestive tract microbiota, and they have been detected by PCR-based methods and cultured

39 from the oral cavity and the stools of apparently healthy individuals (1). More particularly,

40 Methanobrevibacter oralis, Methanobrevibacter smithii and Methanobrevibacter massiliense

41 have been isolated from the oral cavity, whereas M. smithii, Methanosphaera stadtmanae,

42 Methanomassiliicoccus luminiyensis, Methanobrevibacter arboriphilicus, $M$. oralis, Ca.

43 Methanomethylophilus alvus and $\mathrm{Ca}$. Methanomassiliicoccus intestinalis have been isolated

44 from stools (1). Accordingly, we previously showed that virtually all apparently healthy

45 individuals would carry methanogens in the digestive tract microbiota (2). This observation

46 indeed corroborated the pivotal role of methanogens, which detoxify hydrogen produced by

47 bacterial fermentations into methane $(3,4)$.

48 Moreover, methanogens are increasingly implicated in diseases; their presence or absence is

49 associated with dysbioses such as those observed in the gut microbiota in cases of chronic

50 constipation (5), obesity (6) and colonic diseases including ulcerative colitis, Crohn's disease

51 and colorectal cancer (7-9), in the vaginal microbiota in cases of vaginosis $(10,11)$, or their

52 presence in anaerobic pus abscesses such as brain abscesses $(12,13)$ and muscular abscesses

53 (14).

54 As is usual in clinical microbiology, isolation and culture of methanogens is the gold standard

55 to assess the detection of living methanogens in microbiota and in pathological clinical

56 specimens collected by puncture or biopsy (1). The routine application of methanogen

57 culturing is hampered by the fact that methanogens are strictly aero-intolerant microbes and

58 require hydrogen for culturing $(15,16)$. To facilitate the isolation and culture of methanogens

59 from a routine perspective, we previously designed a new process in which methanogens are

60 cultivated in the presence of hydrogen-producing Bacteroides thetaiotaomicron $(17,18)$. 
61 Here, we tested the conditions to replace the biological production of hydrogen with a

62

63

64

chemical production of hydrogen and applied it to isolate methanogens from saliva as a proof-of-concept.

\section{MATERIALS AND METHODS}

Chemical production of hydrogen. The immediate chemical production of hydrogen resulting from the oxidation of iron by a weak acid has long been known (19). We used acetic acid in our experiment because of its ability to oxidize iron, resulting in sufficient production of hydrogen (20). We used two solutions for our experiments, namely, SAB culture broth (21) and distilled water (Fresenius, Bad Homburg voor Hoehe, Germany) with pH values of 7.3 and $\mathrm{pH}$ values of 7 , respectively. We used $5 \mathrm{~mL}$ of each solution in our experiments. The experiments were performed in 10 Hungate tubes (Dominique Dutscher, Brumath, France) for each solution. Iron filings (Amazon, Clichy, France) were used at increasing quantities: $0.5 \mathrm{~g}$ for tube $\mathrm{n}^{\circ} 1,1 \mathrm{~g}$ for tube $\mathrm{n}^{\circ} 2$, and a $0.25 \mathrm{~g}$ increase from tube $\mathrm{n}^{\circ} 3$ to tube $\mathrm{n}^{\circ} 10$. The amount of acetic acid (VWR International, Pennsylvania, USA) used was $100 \mu \mathrm{L}$ for tube $\mathrm{n}^{\circ} 1$ to tube $\mathrm{n}^{\circ} 7$ and $50 \mu \mathrm{L}$ for tube $\mathrm{n}^{\circ} 8$ to tube $\mathrm{n}^{\circ} 10$. We first put the iron filings in the tubes, followed by the solution (SAB broth or water) and then the acetic acid. We used three negative control Hungate tubes, one tube with iron filings in SAB culture medium, another tube with iron filings in distilled water and finally a third tube containing iron filings alone. Gas chromatography was then performed using a Clarus 580 FID chromatograph (PerkinElmer, Villebon-sur-Yvette, France) to measure hydrogen production in the different tubes, and the $\mathrm{pH}$ was monitored (Fisher Scientific, Illkirch, France).

\section{Culture of methanogen strains using the chemical method for hydrogen production. $M$.} oralis CSUR P9633, a human saliva isolate (22), was used for the development of the chemical method. We used the mini-double-chamber flask technique, derived from the one 
87 previously described (21). SAB agar plates (5-cm plates, Fisher Scientific) inoculated with

$88200 \mu \mathrm{L}$ of a M. oralis suspension at $10^{7}$ colony-forming units (CFUs) was placed in the upper

89 compartment of the mini-double-chamber flask, which was sealed with parafilm to ensure an

90 anaerobic atmosphere. We then put $1 \mathrm{~g}$ of iron filings and $100 \mu \mathrm{L}$ of acetic acid in $10 \mathrm{~mL}$ of

91 distilled water in the lower compartment of the mini-double-chamber flask in a

92 microbiological safety station. We used an SAB agar plate inoculated with $200 \mu \mathrm{L}$ of sterile

93 phosphate buffered saline (PBS) (Fisher Scientific) as a negative control. We then incubated

94 the plates at $37^{\circ} \mathrm{C}$ for nine days with visual inspection on day 3, day 6 and day 9

95 postinoculation.

96 Then, the growth of M. smithii CSUR P9632, a human stool isolate, was compared using the

97 chemical method with the reference biological method using a double-chamber system as

98 previously described for the aerobic culture of methanogens (17). For the chemical method,

99 we put $1.5 \mathrm{~g}$ of iron filings and $150 \mu \mathrm{L}$ of acetic acid in $200 \mathrm{~mL}$ of distilled water in the lower

100 compartment instead of Bacteroides thetaiotaomicron, which was used in the biological

101 method. SAB agar plates inoculated with $200 \mu \mathrm{L}$ of a $M$. smithii suspension at $10^{6} \mathrm{CFU}$ or

$102200 \mu \mathrm{L}$ of PBS for the negative controls were placed in the upper compartment.

103 The $\mathrm{pH}$ of the $\mathrm{SAB}$ agar plates was measured at day 3, day 6 and day 9 postinoculation using

$104 \mathrm{pH}$ indicator strips (Macherey-Nagel SARL, Hœrdt, France). We placed the pH indicator

105 strip directly on the SAB agar plate for two minutes. We then took the value corresponding to

106 the color obtained on the strip from the values indicated by the supplier. The $\mathrm{pH}$ and the

107 redox potential of the broth in the lower chamber were measured at day 3, day 6 and day 9

108 postinoculation using the Accumet ${ }^{\mathrm{TM}}$ AE150 apparatus (Fisher Scientific). The probe was

109 rinsed thoroughly with distilled water and then immersed in the lower chamber until the

110 device displayed the value on the reading screen. The rinsing step was performed after each

111 use to avoid any error in reading the device. We used the $\mathrm{pH}$ and redox potential of distilled 
112 water alone and the SAB culture broth alone as controls. We also measured the redox

113 potential of the upper compartment. For the two methods, we used one double-chamber

114 system, in which we put one Petri plate with $10 \mathrm{~mL}$ of distilled water and one Petri plate with

$11510 \mathrm{~mL}$ of the SAB culture broth. These double-chamber systems were then incubated at

$11637^{\circ} \mathrm{C}$, and the redox potential was measured in each of the Petri dishes at day 3, day 6 and

117 day 9 postinoculation. The effectiveness of the two methods for the growth of methanogens

118 was compared by observing the appearance of the first colonies as well as the average

119 number of colonies at day 3, day 6 and day 9 postinoculation. Colonies were confirmed by

120 autofluorescence optical microscopy as follows. Briefly, the colony deposited on a

121 microscopy slide was observed at 63X using an epifluorescence microscope (Leica DMI

1223000 , Wetzlar, Germany) and at 100X magnification using another epifluorescence

123 microscope (Leica DMI 6000). Colonies were enumerated at day 3, day 6 and day 9

124 postinoculation by focusing the image of the entire 5-cm plate on a black background using a

125 portable camera without flash (Lenovo L38011, Beijing, China). Numbered images were

126 analyzed and counted using ImageJ version 8 (https://imagej.nih.gov/ij/download.html)

127 (Wayne Rasband, Java, National Institutes of Health, USA) as follows: each image was

128 imported into Image J software and analyzed in a first step using the blue, diamidino

129 phenylindole filter to normalized the image. In a second step, the normalized image was

130 analyzed using the green fluorescein isothiocyanate filter and saved in the Joint Photographic

131 Experts Group format. We standardized the images using the elliptical selection tabulation of

132 the software. We then proceeded to manually count the enlarged colonies using the

133 magnifying glass and the multipoint tabulation of the software.

135 Comparison of the two methods on saliva samples. The study was previously approved by

136 the Ethics Committee of the IHU Méditerranée infection under n²016-011. After having 
137 informed and collected the consent of the participants, we collected saliva samples from 50

138 people, including 25 tobacco smokers (15 men, 10 women, median age of 30 ) and 25

139 nonsmokers (12 men, 13 women, median age of 28). The collection and processing of

140 samples were performed as previously described (22). We used SAB agar plates inoculated

141 with $200 \mu \mathrm{L}$ of PBS as negative controls and then proceeded in the same manner as described

142 above for the $M$. smithii strain.

144 Molecular analysis. PCR was performed to confirm the identity of the colonies. Colonies

145 were suspended in $200 \mu \mathrm{L}$ of ultrapure water (Fisher Scientific), and a sonication step was

146 performed for 30 minutes. DNA was then extracted with the EZ1 Advanced XL Extraction

147 Kit (QIAGEN, Hilden, Germany) using $200 \mu \mathrm{L}$ as the sample volume and $200 \mu \mathrm{L}$ as the

148 elution volume. Gene amplification and PCR sequencing were performed as previously

149 described (22-24). Real-time PCR analyses were performed as previously described (25).

150

151 RESULTS AND DISCUSSION

152 We report on the use of chemically produced hydrogen for the isolation and culture of 153 methanogens as an alternative to the currently used biological method (17). The method we

154 report is based on the production of hydrogen resulting from the chemical reaction between

155 iron filings and a weak acid such as acetic acid. All reported results were validated by

156 negative controls that remained negative and identification of colonies by autofluorescence 157 and PCR-based analyses.

158 We first determined an optimal balance between the concentration of iron filings and

159 acetic acid to maintain the production of hydrogen over several days at a relative

160 concentration compatible with the isolation and culture of methanogens. These preliminary 
161 experiments enabled the development of a safe and controlled production of hydrogen that

162

163

164

165

166

167

168

169

170

171

172

was shown to be efficient in the culture of $M$. oralis and $M$. smithii reference strains.

In the first step, we measured the average redox potential and $\mathrm{pH}$ values of the various media investigated here. The $\mathrm{pH}$ and redox potential of the controls consisting of distilled water and $\mathrm{SAB}$ broth culture medium were 7 and $+81.3 \mathrm{mV}$ and 7.3 and $-11.9 \mathrm{mV}$, respectively. After incubation at $37^{\circ} \mathrm{C}$ for 3,6 and 9 days, these values were $6.98,6.92$ and 6.87 units and $+64.6 \mathrm{mV},+67.3 \mathrm{mV}$ and $+79.9 \mathrm{mV}$ for distilled water; and $6.83,6.78$ and 6.27 units and $+11.1 \mathrm{mV},+12.9 \mathrm{mV}$ and $+14.8 \mathrm{mV}$ for the $\mathrm{SAB}$ broth culture medium, respectively (Table 1). These results agree with previous observations that most types of water, including tap water and bottled water, are oxidizing agents, and the value of their redox potential is positive, $\geq 20 \mathrm{mV}$ (26). The addition of antioxidants in the $\mathrm{SAB}$ medium makes this culture medium reductive. Then, the $\mathrm{pH}$ and redox potential of distilled water enriched with iron filings and acetic acid (chemical method) were measured at 5.23, 5.16 and 5.08 units and $+109.77 \mathrm{mV},+108.6 \mathrm{mV}$ and $+112.7 \mathrm{mV}$, respectively (Table 2 ). These values were $5.8,5.25$, and 5.24 units and $+113.8 \mathrm{mV},+113.8 \mathrm{mV}$ and $+114.5 \mathrm{mV}$ at day 3 , day 6 and day 9 for the SAB culture medium incubated with $B$. thetaiotaomicron, respectively (Table 2). These data indicated that the cultivation of B. thetaiotaomicron in the SAB medium releases fermenting compounds such as $\mathrm{CO}_{2}$, hydrogen and acetate, which could oxidize the SAB medium (26). Then, we measured these values in the Petri dishes placed in the upper compartment of the double-chamber system, which hosted the methanogen culture (Fig. 1). The redox potential values were $-28.1 \mathrm{mV},-81.2 \mathrm{mV}$ and $-108.9 \mathrm{mV}$ at day 3 , day 6 and day 9 for distilled water, respectively, and $+13.5 \mathrm{mV},-116.5 \mathrm{mV}$ and $-77.5 \mathrm{mV}$ at day 3 , day 6 and day 9 for the SAB culture broth in the chemical method, respectively; and for the biological method, the redox potential values were $-4.5 \mathrm{mV},-66 \mathrm{mV}$ and $-91.6 \mathrm{mV}$ at day 3, day 6 and day 9 for distilled water, respectively, and $+19.1 \mathrm{mV},-7.1 \mathrm{mV}$ and $-34.9 \mathrm{mV}$ at day 
1863 , day 6 and day 9 for the SAB culture broth in the biological method, respectively (Table 3).

187 These data indicated that the hydrogen, being a reducer released into the bottom

188 compartment, reaches the upper compartment and reduces the SAB medium or the water

189 placed into Petri dishes in the upper compartment (27).

190 In a second step, by culturing the reference methanogen strains in the presence of the

191 negative and positive controls, we obtained hydrogen production in all tubes and observed a

192 correlation between hydrogen production and the amount of iron filings used and the acid

193 acetic concentration. The hydrogen production using the chemical method was maintained

194 until day 5 , and the average $\mathrm{pH}$ of the mixture was $4.09 \pm 0.15$ and $4.35 \pm 0.23$ in the SAB

195 culture broth and distilled water, respectively. M. oralis colonies that were confirmed by PCR

196 sequencing were visible on day 3 postinoculation using the chemical method (Supplementary

197 Fig. 1). The negative control consisting of PBS inoculation instead of M. oralis remained

198 negative.

199 To compare the chemical method with the biological method, M. smithii was

200 inoculated in SAB agar plates using both methods. Using the chemical method, inoculation of

201 SAB agar plates with $M$. smithii yielded colonies as early as day 3 postinoculation, whereas

202 they appeared on day 9 using the biological method (Supplementary Fig. 1, Fig. 2). All

203 negative controls remained negative. The colonies were confirmed using PCR sequencing

204 and real-time PCR. The average number of colonies of $M$. smithii was significantly higher

205 using the chemical method than the biological method, regardless of the day of follow-up

206 (Student's test, $\mathrm{p}<0.0001, \mathrm{p}<0.001$ and $\mathrm{p}=0.0001$ for day 3 , day 6 and day 9

207 postinoculation, respectively) (Table 4). Moreover, cultures of these two strains were

208 obtained in three days rather than in nine days using the biological method (17). In addition,

209 the chemical method was easier to set up than the biological method because it incorporated

210 distilled water instead of the SAB culture broth used in the chemical method, in contrast to 
211 the biological method in which only the SAB culture broth can be used (Supplementary Table

212 1). Additionally, the biological method requires maintaining a B. thetaiotaomicron culture in

213 the exponential growth phase to release efficient hydrogen production, which is not the case

214 for the chemical method.

215 Finally, we compared the chemical method with the biological method for isolating

216 methanogens from 50 saliva samples collected from 50 individuals after 9 days of incubation.

217 The number of positive samples was $6 / 50(12 \%)$ at day 3 postinoculation using both methods,

218 and one additional saliva sample was positive at day 6 , so that a total of $7 / 50(14 \%)$ were

219 positive using both methods. No additional sample was positive at day 9 postinoculation,

220 regardless of the culture method. In these 7 culture-positive saliva samples, the average

221 number of colonies at day 6 and day 9 postinoculation was significantly higher using the

222 biological method than using the chemical method (Student's test, $\mathrm{p}=0.005$ and $\mathrm{p}=0.04$ ),

223 whereas there was no significant difference in the average number of colonies on day 3

224 (Student's test, $\mathrm{p}=0.09)$ (Table 4). Cultures from 6/25 $(24 \%)$ of the samples from tobacco

225 smokers yielded colonies as early as day 3 in both methods versus 1/25 (4\%) of the samples

226 from nonsmokers at day 6 in both methods. Colonies yielded autofluorescence in both

227 methods (Fig. 2 and Fig. 3), whereas no colony or autofluorescence was observed in the

228 negative controls. PCR sequencing and real-time PCR identified three $M$. oralis-positive and

229 three M. smithii-positive samples in the tobacco smoker samples and one $M$. oralis-positive

230 sample in one nonsmoker sample. Applying the chemical method for the isolation of $M$.

231 oralis from saliva samples yielded significantly more colonies more rapidly than the

232 biological method used in parallel. We hypothesized that the chemical method allowed

233 mastering the kinetics of hydrogen production in such a way to optimize the atmosphere in

234 the double-chamber system used in our experiments. These results show that the chemical

235 method is more effective than the biological method for the rapid culture of methanogens. 
236 Accordingly, the observation that almost a quarter of the saliva samples grew M. oralis and

237 M. smithii is in agreement with a previously published report (22). Interestingly, we

238 confirmed that methanogens are more prevalent in the saliva from tobacco smokers than in

239 the saliva collected from nonsmokers. These results are consistent with those found

240 previously (22), in which there was also a predominance of $M$. oralis over $M$. smithii in saliva

241 samples. These observations also confirm that $M$. oralis is the most prevalent methanogen in

242 the oral cavity. We measured the redox potential in the culture using the chemical method to

243 monitor whether this chemical reaction created anaerobiosis. Our results indicate that this

244 chemical reaction is reducing and confirm that acetic acid is an efficient oxidizer of iron

245 filings (20). The $\mathrm{pH}$ of the agar plates remained neutral throughout the inoculation period

246 both in the chemical method and the biological method; this observation suggests that the

247 acidity produced in the lower compartment of the double-chamber device in the chemical

248 method does not reach the upper compartment.

249 The present study highlighted some advantages of the chemical method over the

250 biological method (Supplementary Table 1). The chemical method is simple to set up, the

251 hydrogen production is controlled so that the time of appearance of the first colonies is fast

252 due to the immediate hydrogen production, and this chemical method can be done with both

253 distilled water and SAB culture broth. Given the simplicity, speed and efficiency of this

254 chemical method of hydrogen production, it could replace the biological method

255 incorporating B. thetaiotaomicron in the isolation and culture of methanogens. Using the

256 chemical method will ease the routine isolation and culture of methanogens in microbiology

257 laboratories to ease and speed the isolation and culture of these opportunistic pathogens (1). 


\section{ACKNOWLEDGEMENTS}

261 COG and GG benefit from Ph.D. grants from the Fondation Méditerranée Infection,

262 Marseille, France. This work was supported by the French Government under the

263 «Investissements d'avenir» (Investments for the Future) program managed by the Agence

264 Nationale de la Recherche (ANR, fr: National Agency for Research), (reference:

265 Méditerranée Infection 10-IAHU-03). This work was supported by Région Sud (Provence

266 Alpes Côte d'Azur) and European funding FEDER PRIMMI. 


\section{REFERENCES}

269 1. Sogodogo E, Drancourt M, Grine G. 2019. Methanogens as emerging pathogens in $270 \quad$ anaerobic abscesses. Eur J Clin Microbiol Infect Dis 2:19-25.

271 2. Dridi B, Henry M, El Khéchine A, Raoult D, Drancourt M. 2009. High prevalence of 272 Methanobrevibacter smithii and Methanosphaera stadtmanae detected in the human gut 273 using an improved DNA detection protocol. PLoS ONE 4:e7063.

274 3. Deppenmeier U, Lienard T, Gottschalk G. 1999. Novel reactions involved in energy 275 conservation by methanogenic archaea. FEBS Lett 457:291-297.

276 4. Deppenmeier U. 2002. Redox-driven proton translocation in methanogenic Archaea. $277 \quad$ Cell Mol Life Sci 59:1513-1533.

278 5. Chatterjee S, Park S, Low K, Kong Y, Pimentel M. 2007. The Degree of breath methane 279 production in IBS correlates with the severity of constipation. AJG 102:837-841.

280 6. Zhang H, DiBaise JK, Zuccolo A, Kudrna D, Braidotti M, Yu Y, Parameswaran P, 281 Crowell MD, Wing R, Rittmann BE, Krajmalnik-Brown R. 2009. Human gut microbiota in obesity and after gastric bypass. Proc Natl Acad Sci 106:2365-2370.

7. Piqué JM, Pallarés M, Cusó E, Vilar-Bonet J, Gassull MA. 1984. Methane production and colon cancer. Gastroenterology 87:601-605. 
288 9. Ghavami SB, Rostami E, Sephay AA, Shahrokh S, Balaii H, Aghdaei HA, Zali MR. 2018. Alterations of the human gut Methanobrevibacter smithii as a biomarker for inflammatory bowel diseases. Microb Patho 117:285-289.

10. Belay N, Mukhopadhyay B, Conway de Macario E, Galask R, Daniels L. 1990. Methanogenic bacteria in human vaginal samples. J Clin Microbiol 28:1666-1668. Methanobrevibacter smithii in vaginal samples collected from women diagnosed with bacterial vaginosis. Eur J Clin Microbiol Infect Dis 21:34-36.

12. Drancourt M, Nkamga VD, Lakhe NA, Régis J-M, Dufour H, Fournier P-E, Bechah Y, Michael Scheld W, Raoult D. 2017. Evidence of Archaeal methanogens in brain abscess. Clin Infect Dis 65:1-5.

13. Nkamga VD, Lotte R, Chirio D, Lonjon M, Roger P-M, Drancourt M, Ruimy R. 2018. Methanobrevibacter oralis detected along with Aggregatibacter actinomycetemcomitans in a series of community-acquired brain abscesses. Clin Microb Infect 24:207-208. smithii and Bacteroides thetaiotaomicron cultivated from a chronic paravertebral muscle abscess. Clin Microb Infect 22:1008-1009. diversity of methanogenic Archaea. Anaerobe 6:205-226. 
309 17. Khelaifia S, Lagier J-C, Nkamga VD, Guilhot E, Drancourt M, Raoult D. 2016. Aerobic

310 culture of methanogenic archaea without an external source of hydrogen. Eur J Clin

$311 \quad$ Microbiol Infect Dis 35:985-991.

312 18. Traore SI, Khelaifia S, Armstrong N, Lagier JC, Raoult D. 2019. Isolation and culture of

313 Methanobrevibacter smithii by co-culture with hydrogen-producing bacteria on agar

314 plates. Clin Microb Infect 12:45-47.

315 19. Boddien A, Loges B, Gärtner F, Torborg C, Fumino K, Junge H, Ludwig R, Beller M.

316 2010. Iron-catalyzed hydrogen production from formic acid. J Am Chem Soc 132:8924-

$317 \quad 8934$.

318 20. Crouzet C, Brunet F, Recham N, Findling N, Lanson M, Guyot F, Ferrasse J-H, Goffé

$319 \quad$ B. 2017. Hydrogen production by hydrothermal oxidation of $\mathrm{FeO}$ under acidic

$320 \quad$ conditions. Inter J Hyd E 42:795-806.

321

322

21. Khelaifia S, Raoult D, Drancourt M. 2013. A Versatile Medium for Cultivating Methanogenic Archaea. PLoS ONE 8:e61563.

22. Grine G, Terrer E, Boualam MA, Aboudharam G, Chaudet H, Ruimy R, Drancourt M. 2018. Tobacco-smoking-related prevalence of methanogens in the oral fluid microbiota. Sci Rep 8:23-26. 
329 24. Nkamga VD, Huynh HTT, Aboudharam G, Ruimy R, Drancourt M. 2015. Diversity of

330 human-associated Methanobrevibacter smithii isolates revealed by multispacer sequence

$331 \quad$ typing. Curr Microbiol 70:810-815.

332 25. Bringuier A, Khelaifia S, Richet H, Aboudharam G, Drancourt M. 2013. Real-time PCR

333 quantification of Methanobrevibacter oralis in periodontitis. J Clin Microbiol 51:993-

334994.

335 26. Adamberg S, Tomson K, Vija H, Puurand M, Kabanova N, Visnapuu T, Jaugi E, Alame

336 T, Adamberg K. 2014. Degradation of fructans and production of propionic acid by

337 Bacteroides thetaiotaomicron are enhanced by the shortage of amino acids. Front Nut

$338 \quad 1: 54-60$.

339 27. Thauer RK, Kaster A-K, Seedorf H, Buckel W, Hedderich R. 2008. Methanogenic

340 archaea: ecologically relevant differences in energy conservation. Nat Rev Microbiol

$341 \quad 6: 579-591$. 
bioRxiv preprint doi: https://doi.org/10.1101/734210; this version posted August 14,2019 . The copyright holder for this preprint (which was not certified by peer review) is the author/funder. All rights reserved. No reuse allowed without permission.

\section{TABLES}

344 Table 1. Controls of $\mathrm{pH}$ and redox potential.

\begin{tabular}{l|cccccccc}
\multicolumn{1}{c}{ Controls } & \multicolumn{4}{c}{ Redox potential $(\mathrm{mV})$} & \multicolumn{3}{c}{$\mathrm{pH}$} \\
\cline { 2 - 9 } & D0 & D3 & D6 & D9 & D0 & D3 & D6 & D9 \\
Distilled water & +81.3 & +64.6 & +67.3 & +79.9 & 7 & 6.98 & 6.92 & 6.87 \\
SAB medium & -8.7 & +11.1 & +12.9 & +14.8 & 7.3 & 6.83 & 6.78 & 6.27
\end{tabular}


bioRxiv preprint doi: https://doi.org/10.1101/734210; this version posted August 14,2019 . The copyright holder for this preprint (which was not certified by peer review) is the author/funder. All rights reserved. No reuse allowed without permission.

347 Table 2. $\mathrm{pH}$ and redox potential of the lower compartment.

348

Methods

Chemical method

Biological method

\begin{tabular}{|cccccc|}
\multicolumn{4}{c}{ Redox potential $(\mathrm{mV})$} & \multicolumn{3}{c}{$\mathrm{pH}$} \\
$\mathrm{D} 3$ & $\mathrm{D} 6$ & $\mathrm{D} 9$ & $\mathrm{D} 3$ & $\mathrm{D} 6$ & $\mathrm{D} 9$ \\
+109.77 & +108.6 & +112.7 & 5.23 & 5.16 & 5.08 \\
+113.8 & +113.8 & +114.5 & 5.8 & 5.25 & 5.24
\end{tabular}

349 
350 Table 3. Redox potential of the upper compartment.

Redox potential (mv)

Distilled water

SAB medium
Chemical method

Biological method

\begin{tabular}{cccccc}
\hline D3 & D6 & D9 & D3 & D6 & D9 \\
-28.1 & -81.2 & -108.9 & -4.5 & -66 & -91.6 \\
+13.5 & -116.5 & -77.5 & +19.1 & -7.1 & -34.9
\end{tabular}

351

352 
353 Table 4. Comparison of the two methods according to the average of the colonies counted by

354 ImageJ.

\begin{tabular}{r|lllllllll} 
Methods & Chemical method & \multicolumn{7}{c}{ Biological method } \\
\cline { 2 - 10 } & D3 & D6 & D9 & D3 & D6 & D9 & D3 & D6 & D9 \\
M. smithii & $377 \pm 72$ & $659 \pm 168$ & $1116 \pm 193$ & 0 & 0 & $436 \pm 74$ & $<0.0001$ & $<0.001$ & 0.0001 \\
& & & & & & & & & \\
Saliva & $190 \pm 90$ & $509 \pm 133$ & $700 \pm 232$ & $305 \pm 139$ & $722 \pm 96$ & $936 \pm 136$ & 0.09 & 0.005 & 0.04 \\
samples & & & & & & & & & \\
$\begin{array}{r}\text { Negative } \\
\text { controls }\end{array}$ & 0 & 0 & 0 & 0 & 0 & 0 & NA & NA & NA \\
NA : Not adapted & & & & & & & &
\end{tabular}




\section{FIGURES}

358 Figure 1. Aerobic culture of methanogens in a double-chamber system on agar plates using

359 either a microbiological method for hydrogen production (red arrow) or a chemical method

360 for hydrogen production investigated here (blue arrow).

361 Figure 2. (A) Fluorescence emitted by M. smithii by applying the blue filter set. (B)

362 Fluorescence emitted by $M$. smithii by applying the green filter set.

363 Figure 3. (A) Fluorescence emitted by M. oralis by applying the blue filter set. (B)

364 Fluorescence emitted by $M$. oralis by applying the green filter set.

365

366

367

368

369

370

371

372

373

374

375

376

377

378

379

380

381

382

383

384

385

386

387

388

389 

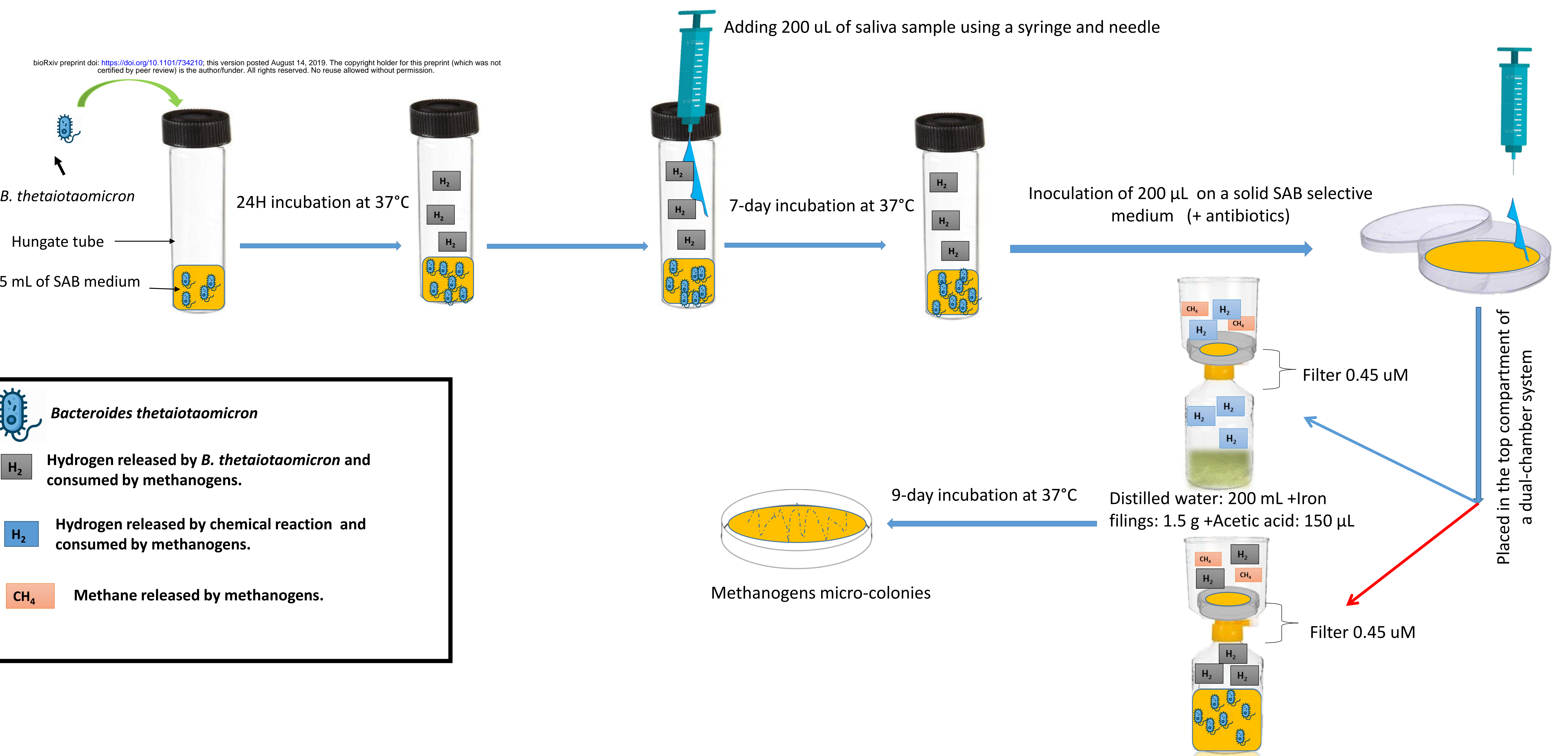
FIGURE 2

A

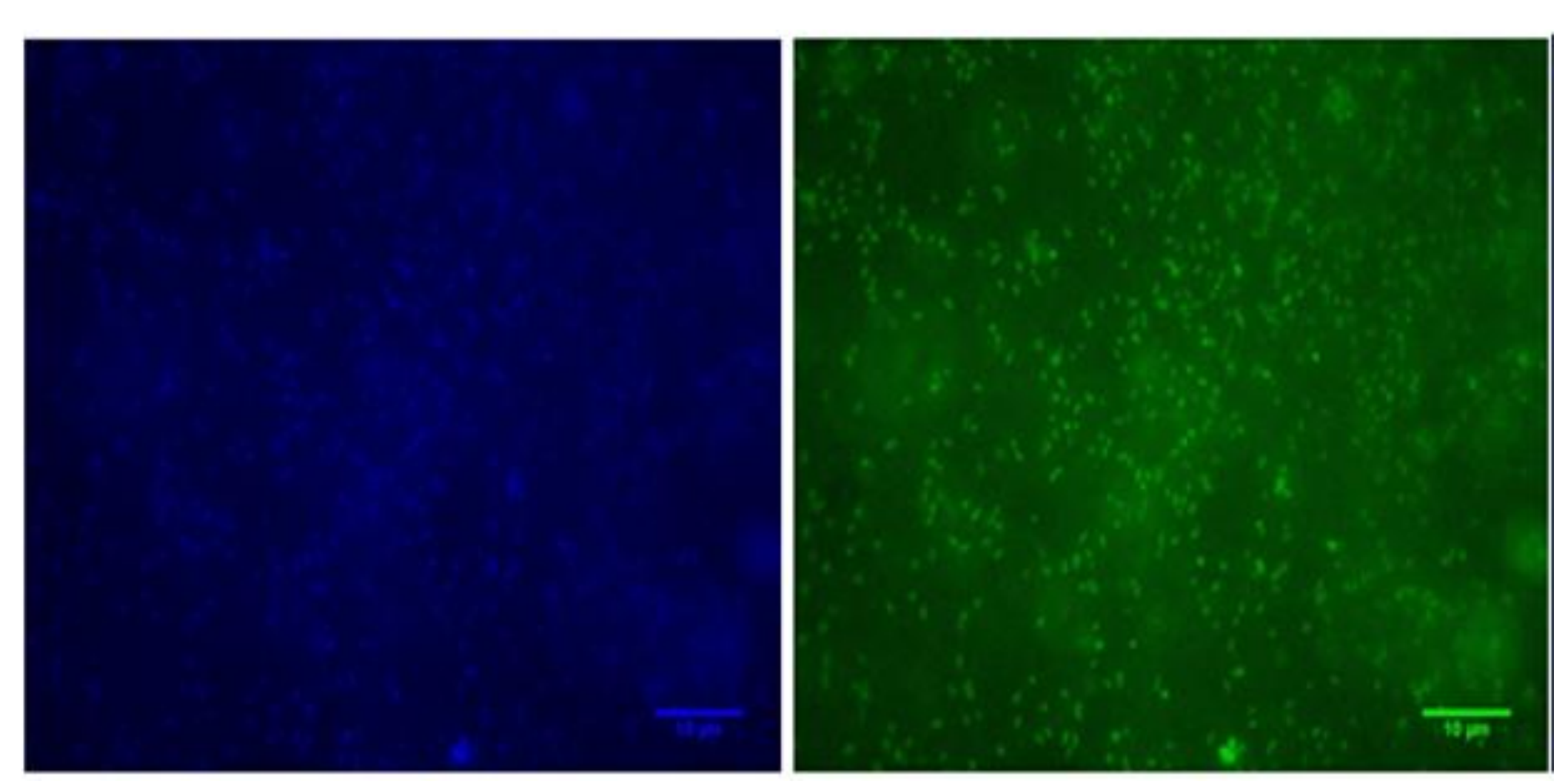

B 


\section{FIGURE 3}

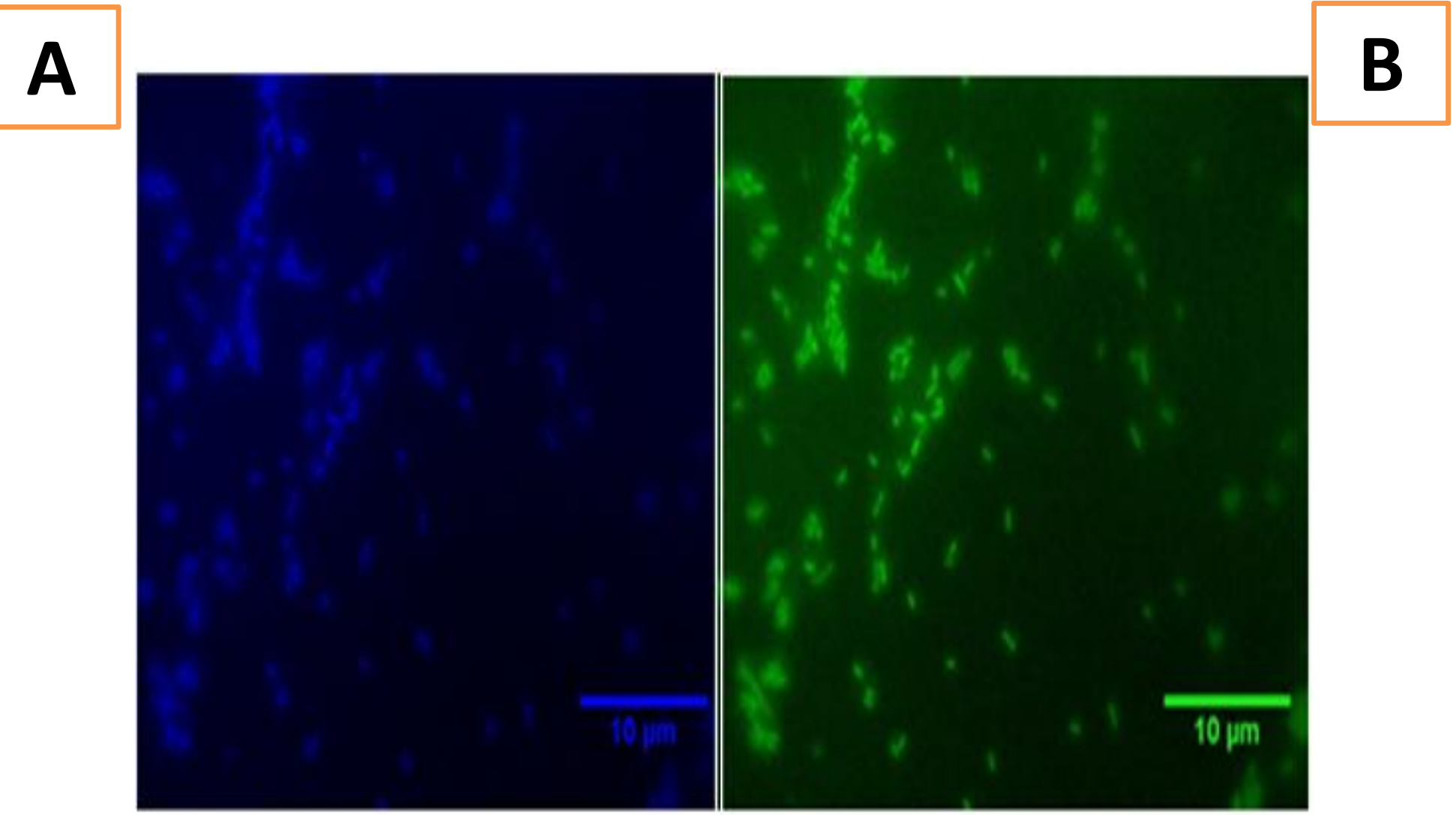

